\title{
Prostatic adenocarcinoma with renal metastases in a dog diagnosed by contrast enhanced ultrasound - case report
}

\author{
Diana Feier ${ }^{2}$, Mircea Mircean ${ }^{1}$, Robert Cristian Purdoiu ${ }^{1}$, Adrian Gal ${ }^{1}$, Ionel Papuc ${ }^{1}$, \\ Radu Badea ${ }^{2}$ \\ ${ }^{1}$ University of Agricultural Sciences and Veterinary Medicine, Faculty of Veterinary Medicine, Cluj Napoca, \\ Romania \\ ${ }^{2}$ University of Medicine and Pharmacy "Iuliu Hațieganu”, Department of Ultrasound, Cluj Napoca, Romania
}

Received December 28, 2011

Accepted October 23, 2012

\begin{abstract}
Prostate cancer in dogs belongs to the category of diseases that affect mainly elderly animals. Due to low frequency and slow development of this disease, the diagnosis and staging of these tumours present a challenge. The aim of this study was to highlight the role of contrast enhanced ultrasound in the diagnosis and staging in a case report of prostate adenocarcinoma in Rottweiler aged 7 years. Conventional ultrasound examination of the dog revealed a profoundly altered structure of the prostate, the presence of masses in both the kidney and liver parenchyma with hepatic veins consistent with turgor, and also a hyperechoic content of the gallbladder. Optimization of the examination with contrast substance revealed a vascular pattern of malignancy both in the prostate and kidney, and has excluded any bile and kidney determinations. Pathological examination revealed a primary tumour in the form of an anaplastic prostate adenocarcinoma with multiple secondary determinations in the lung, kidney and bone. Ultrasound enhanced with intravenously administered contrast substance is an optimal imaging technique that provides real-time information on the vascular space replacement formations behaviour in prostate and kidney, with a role in the diagnosis and staging of prostate tumours.
\end{abstract}

Prostate gland tumour, tumour enhancement pattern, canine, kidney, echography

Prostate disease in dogs is a common category of diseases in current clinical practice (Smith 2008). Canine prostate adenocarcinoma is rare; however, other domestic animals are affected in exceptionally few cases (MacLachLan and Kennedy 2002).

Detection of prostate tumour formations in animals and humans can be done with high diagnostic accuracy using ultrasound-imaging procedures (Halpern 2006; Wink et al. 2007). Characteristics of malignancy are nonspecific though, making it difficult to differentiate them (Matton and Nyland 2002). In dogs, gray scale ultrasound seems to be too rough to establish a definite diagnosis, problems are apparent in differentiating between different types of prostate inflammation (Pacliková et al. 2007). The possibility of real time analysis of the degree of contrast in the tumour makes the procedure of contrast enhanced ultrasonography (CEUS - contrast medium applied to traditional ultrasonography) to have added value in the diagnosis of tumour diseases in dogs. Intratumoural behavior of contrast directly correlates with the nature of the tumour, which may constitute the basis for noninvasive diagnosis. When appropriate, there is the possibility to identify the strongest injurious focus, which allows targeted biopsy and thereby accurate diagnosis (Sparchez et al. 2010). The use of CEUS significantly reduces diagnostic errors that can occur in gray scale ultrasound or in colour Doppler.

Ultrasound examination is at the forefront of these procedures as a widespread, cheap, and easy to do method. The dynamic nature of ultrasound procedure is essential for this investigation, playing a fundamental role in the clinical examination.

Address for correspondence:

Robert Cristian Purdoiu

Faculty of Veterinary Medicine

University of Agricultural Sciences and Veterinary Medicine

Calea Manastur, Nr. 3-5, 400 372, Cluj Napoca, Romania

Phone: +40 745085279

E-mail: robcry999@yahoo.com

http://actavet.vfu.cz/ 
This study presents a case report of prostatic adenocarcinoma with renal metastases in a dog diagnosed by contrast enhanced ultrasound compared to gray scale ultrasound and Doppler.

\title{
Materials and Methods
}

\begin{abstract}
Case report
A male Rottweiler, seven years old, underweight with body mass $27 \mathrm{~kg}$, was brought to consultation for progressive weight and appetite loss, installed insidiously in about 6 weeks.

Method used

Contrast enhanced ultrasonography examination was performed using low acoustic power (mechanical index $<0.12$ ) after injection of a contrast agent of the second generation, SonoVue (Bracco, Milan, Italy). Two ml of the contrast agent were injected i.v. followed by $10 \mathrm{ml}$ of saline. The examination protocol used the contrast software of the machine, pulse inversion mode ("pulse inversion"). The examination was dynamic, following tumours in a successive way, in the arterial phase (within 10-30 s after injection), venous phase (30-120 s) and late phase (more than $120 \mathrm{~s}$ to the strict liver, in order to detect any metastasis at this level). The information obtained was stored in the form of video clips that allowed subsequent analysis and post processing. The main ultrasound features, both in gray scale and postcontrast were evaluated according to guidelines developed by the European Federation of Societies for Ultrasound in Medicine and Biology (Claudon et al. 2008).

Tumour ultrasound appearance was based on anatomical criteria: presence of structural changes, with a different pattern than that of healthy parenchyma, surrounding structures and even invading them, the presence of exaggerated circulation which is spatially disordered as you can see in the colour coded Doppler exploration. For gray scale ultrasound examination of the liver and kidney we used a convex probe at 4.7-5 $\mathrm{MHz}$ (dynamic range $1-7 \mathrm{MHz}$ ) and a linear probe at $11 \mathrm{MHz}$ (dynamic range 7-14 MHz). For colour Doppler examination the frequency used was 3.6 MHz. The examinations were conducted under neuroleptanalgesia (ketamine at a dose of $2 \mathrm{mg} / \mathrm{kgw}+$ xylazine at a dose of $0.3 \mathrm{mg} / \mathrm{kgw}$ i.m., manufactured by Animal Health GMP, Netherland).
\end{abstract}

\section{Results}

Clinical and subsequent paraclinical examination revealed a malignant impregnation syndrome with the emaciation of skeletal muscles and hair root damage, polypnea evident at rest with severe anaemia (red blood cell count $2.8 \times 10^{6} / \mu \mathrm{l}$ compared to normal value of 5.4-7.8 $\times 10^{6} / \mu \mathrm{l}$ and haemoglobin $10.2 \mathrm{~g} / \mathrm{dl}$ compared to normal value of $13-20 \mathrm{~g} / \mathrm{dl}$ ) with neutrophilia syndrome (neutrophils $81 \%$, normal value $60-70 \%$ ), renal function deterioration with nitrogen retention syndrome, hepatic cholestasis, hypoglycaemia (glucose $57.4 \mathrm{mg} / \mathrm{dl}$, normal value $70-120 \mathrm{mg} / \mathrm{dl}$ ), hypokalaemia (potassium $2.98 \mathrm{mEq} / \mathrm{l}$, normal value $3.5-5.5 \mathrm{mEq} / \mathrm{l}$ ) and hypercalcaemia (calcium $12.8 \mathrm{mg} / \mathrm{dl}$, normal value 9.00$11.50 \mathrm{mg} / \mathrm{dl}$ ) (reference for ranges of normal values according to Vaden et al. 2009). Chest radiography revealed multiple nodular opacities in both lungs, with secondary pulmonary determinations, but no specific features.

Gray scale ultrasound examination performed by first intention revealed multiple tumours present in both kidneys and the prostate with heterogeneous characters, nonspecific for malignancy, without being able to differentiate the malignant primary tumour and the secondary possible determinations. Despite difficult examination conducted under neuroleptanalgesia, the pelvic ultrasound exam revealed a canine prostate of enlarged size of $78 \times 90 \mathrm{~mm}$, unhomogenous, by the presence of nodular formations, fully delineated and hypoechogenic compared to the surrounding parenchyma. Bilateral in the renal lodge, tumour transformation of both kidneys was revealed by the presence of numerous nodular formations, heterogeneous, with a hypoechogenic centre.

In the Doppler examination of the prostate, the formation seemed to be poorly vascularized, with obvious vascular signal around the tumour (Plate VII, Fig. 1). The Doppler examination of the renal lodge show peripheral vascularization and changes in the renal vascular pattern (Plate VII, Fig. 2).

The ultrasound appearance of the liver showed no significant changes in the conventional examination in gray scale or colour Doppler, except for markedly increased gallbladder 
volume, with a solid content, hyperechogenic, without obscurity and without vascular signal detected both intra- or pericavitary. It was impossible to differentiate benign from malignant nature of the content of bile.

Due to lack of specificity of the characteristics described, conventional ultrasound examination could not specify the location of the primary tumour, so the diagnosis was completed by CEUS. Contrast enhanced ultrasonography examination of the prostate lodge revealed an early capture of the contrast substance during the arterial and an early wash during the venous period, behaviour suggestive for tumours (Plate VIII, Fig. 3). A time to peak (TTP) of $24.5 \mathrm{~s}$ and peak intensity perfusion (PPI) of $23 \%$ were determined. Bilateral CEUS examination of tumours in the kidney revealed alterations in the physiological kidney structure by the presence of hypervascular formations with intense early capture in the arterial period and early wash during the venous period (Plate VIII, Fig. 4). Haemodynamic behaviour is suggestive of secondary metastases, with a TTP of $15 \mathrm{~s}$ and PPI of $16 \%$. The CEUS examination of the liver and bile excluded the presence of secondary metastases at this level and confirmed the diagnosis of biliary sludge (Plate IX, Fig. 5). Biliary sludge was diagnosed in gray scale ultrasound and was initially mistaken for a metastasis in the gall bladder. This abundant muddy-looking sludge is most likely due to vascular congestion in the liver and loss of appetite.

Because of the primary tumour location in the prostate with multiple secondary metastases, euthanasia was chosen. Autopsy confirmed the location of the primary tumor in the prostate as adenocarcinoma with high-grade anaplastic alterations (Plate IX, Fig. 6) and multiple secondary metastases in the kidneys, lungs and bones.

In our case, the initial diagnosis of the prostate tumour and renal masses was based on the criteria listed above. But the main feature, which is directly related to the nature of the tumour, was the peripheral capture mode both central and perilesional as resulted after i.v. administration of the contrast agent combined with harmonic examination. The time required to achieve maximum contrast in the tumours ranged between 20 to $25 \mathrm{~s}$ for the prostate and 14 to $19 \mathrm{~s}$ for CEUS examination of kidney metastases.

\section{Discussion}

Although benign prostate tumours in mammals are a common diagnosis in veterinary clinical practice, the overall incidence of prostate cancer is low in animals, with a frequency of 5-7\% in dogs (Memon 2007; Smith 2008). In a European study conducted on 15000 dogs, 431 dogs were diagnosed with diseases of the prostate, of which only 56 individuals had a carcinoma of the prostate (Teske 2002).

Common diagnostic algorithm requires an assessment of initial imaging to detect any prostate, kidney and spleen tumour. General imaging appearance of these tumours is nonspecific, benign and malignant tumours often have the same model (Marret et al. 2004; Claudon et al. 2008; Vignoli et al. 2011). A differential diagnosis between benign and malignant tumours, the primary and metastatic is always required, often being necessary to perform an ultrasound guided puncture (Leav and Ling 1968; Cornell et al. 2002; Lai et al. 2008). Liver metastases are rare in prostate adenocarcinoma (Leav and Ling 1968).

The particularity of the presented case lies in the spectrum of characteristics described by contrast enhanced ultrasonography in diagnosis orientation toward the primary tumour in the prostate and secondary injuries.

The time required to achieve maximum contrast in malignancies of the prostate is $26.9 \pm 4.8 \mathrm{~s}$ (Vignoli et al. 2011); we obtained a value ranging between 20 to $25 \mathrm{~s}$ for prostate and 14 to $19 \mathrm{~s}$ for CEUS examination of kidney metastases. The appearance of prostate tumour pathology detected by us, after the classification of Leav and Ling (1968), belongs to the intra-alveolar proliferative anaplastic adenocarcinoma (type I); 
secondary metastases of this type of adenocarcinoma are commonly found in the lungs, chest wall, splenic metastases are rare; and renal metastases were found in adenocarcinoma of the prostate anaplastic type II (acinary) and III (syncytial).

Because the epithelial cell differentiation model of human prostate proposed by Isaacs and Coffey (1989) can be applied to dogs (Lai et al. 2008; LeRoy and Northrup 2009), we believe that intravenously administered ultrasound contrast agents can be used in the animal experimental model to study the malignant and benign prostate disease in humans.

The case presented revealed a clinical picture suggestive of a malignant impregnation syndrome, with a starting point for prostate adenocarcinoma with metastases in several organs, diagnosed with contrast enhanced ultrasonography and conventional ultrasound, pathologically confirmed. The main characteristic of prostate tumour transit by the contrast administered i.v., was the peripheral and central perilesional capture. Diagnosis was established by pathological examination. The spectrum of characteristics described by contrast enhanced ultrasonography with clinical and biochemical indicators oriented us towards the primary tumour diagnosis in the prostate and secondary injuries. The ultrasound contrast agent SonoVue was well tolerated by dog, the action on physiological indicators was minimal. Ultrasound with contrast agents is an interesting and promising development for the diagnosis and especially characterization of tumour diseases in dogs.

\section{Acknowledgements}

We address thanks to Dr. B. Niculescu (Medist SRL Romania) for the insurance of the infrastructure needed for the procedure.

\section{References}

Claudon M, Cosgrove D, Albrecht T, Bolondi L, Bosio M, Calliada F, Correas JM, Darge K, Dietrich C, D’Onofrio M, Evans DH, Filice C, Greiner L, Jäger K, Jong N de, Leen E, Lencioni R, Lindsell D, Martegani A, Meairs S, Nolsøe C, Piscaglia F, Ricci P, Seidel G, Skjoldbye B, Solbiati L, Thorelius L, Tranquart F, Weskott HP, Whittingham T 2008: Guidelines and good clinical practice recommendations for contrast enhanced ultrasound (CEUS). Ultraschall Med 29: 28-44

Cornell KK, Bostwick DG, Cooley DM, Hall G, Harvey HJ, Hendrick MJ, Pauli BU, Render JA, Stoica G, Sweet DC, Waters DJ 2000: Clinical and pathologic aspects of spontaneous canine prostate carcinoma: A retrospective analysis of 76 cases. Prostate 45: 173-183

Halpern EJ 2006: Contrast-enhanced ultrasound imaging of prostate cancer. Rev Urol 8 (Suppl 1): S29-S37

Isaacs JT, Coffey DS 1989: Etiology and disease process of benign prostatic hyperplasia. Prostate Suppl 2: 33-50

Lai CL, van den Ham R, van Leenders G, van der Lugt J, Teske E 2008: Comparative characterization of the canine normal prostate in intact and castrated animals. Prostate 68: 498-507

Leav I, Ling G V 1968: Adenocarcinoma of the canine prostate. Cancer 22: 1329-1345

LeRoy BE, Northrup N 2009: Prostate cancer in dogs: Comparative and clinical aspects. Vet J 180: 149-162

MacLachlan NJ, Kennedy PC 2002: Tumors of the genital systems. In: Meuten DJ (4 ${ }^{\text {th }}$ ed.): Tumors in domestic animals. Iowa State University Press, Ames, pp. 568-570

Marret H, Sauget S, Giraudeau B, Brewer M, Moore JR, Body G, Tranquart F 2004: Contrast-enhanced sonography helps in discrimination of benign from malignant addnexal masses. J Ultras Med 23: 1629-1639

Mattoon JS, Nyland TG 2002: Prostate and testes. In: Nyland TG, Mattoon JS (2 ${ }^{\text {nd }}$ eds), Small animal diagnostic ultrasound. W.B. Saunders Company, Philadelphia, pp. 250-266

Memon MA 2007: Common causes of male dog infertility. Theriogenology 68: 322-328

Paclíková K, Kohout P, Vlašín M 2007: Catheter biopsy as a useful tool to establish an early diagnosis for canine prostatic disorders. ACTA Vet Brno 76: 475-485

Smith J 2008: Canine prostatic disease: a review of anatomy, pathology, diagnosis, and treatment. Theriogenology 70: 375

Sparchez Z, Radu P, Zaharia T, Kacso G, Grigorescu I, Badea R 2010: Contrast enhanced ultrasound guidance: a new tool to improve accuracy in percutaneous biopsies. Med Ultrason 12: 133-138

Teske E, Naan EC, Van Dijk EM, Van Garderen E, Schalken JA 2002: Canine prostate carcinoma: epidemiological evidence of an increased risk in castrated dogs. Mol Cell Endocrinol 197: 251-255

Vaden SL, Knoll JS, Smith FWK Jr, Tilley LP 2009: Blackwell's Five-Minute Veterinary Consult: Laboratory Tests and Diagnostic Procedures: Canine and Feline. Blackwell Publishing, Ames, Iowa, USA

Vignoli M, Russo M, Catone G, Rossi F, Attanasi G, Terragni R, Saunders JH, England GCW 2011: Assessment of vascular perfusion kinetics using contrast-enhanced ultrasound for the diagnosis of prostatic disease in dogs. Reprod Domest Anim 46: 209-213

Wink MH, de la Rosette JJMCH, Grimbergen CA, Wijkstra H 2007: Transrectal contrast enhanced ultrasound for diagnosis of prostate cancer. World J Urol 25: 367-373 
Plate VII

Feier D. et al.: Prostatic... pp. 87-90

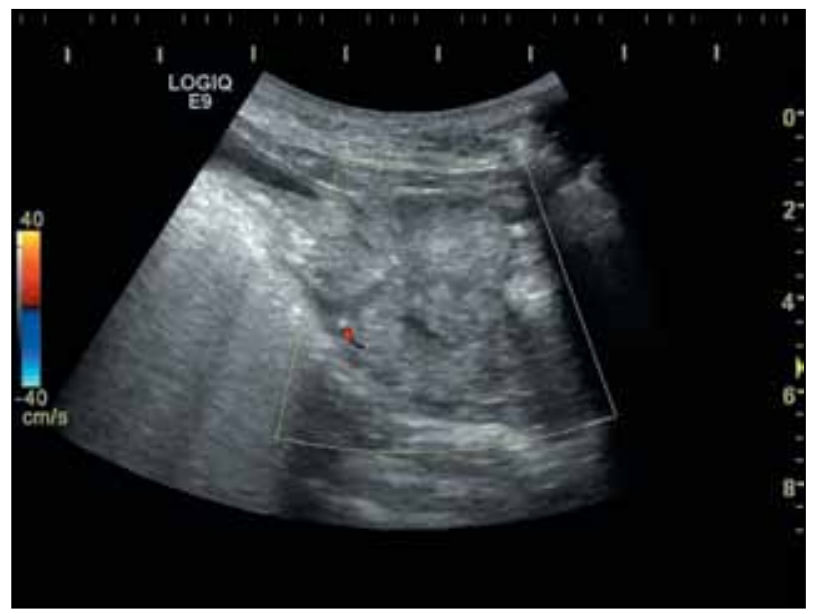

Fig. 1. Conventional ultrasound B mode shows a structure of canine prostate profoundly altered by the presenceof well-defined nodular formations $(*)$. Heterogenic, poor peripheral vascularization (arrow).

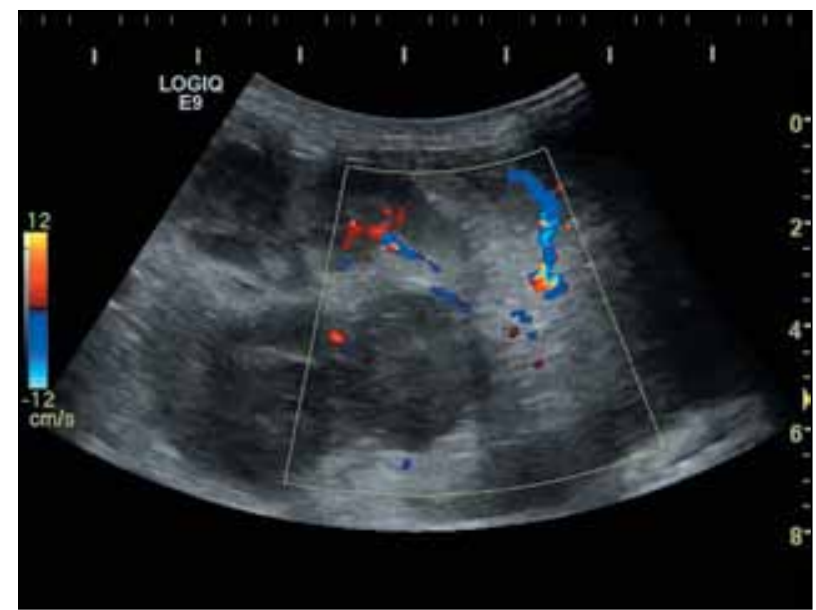

Fig. 2. Profoundly altered kidney structure by the presence of multiple nodular formations, round, heterogenic, located at the junction of medulla and renal cortex (*). Peripheral vascular formations signs and distortion of normal renal vascularization (arrows). 


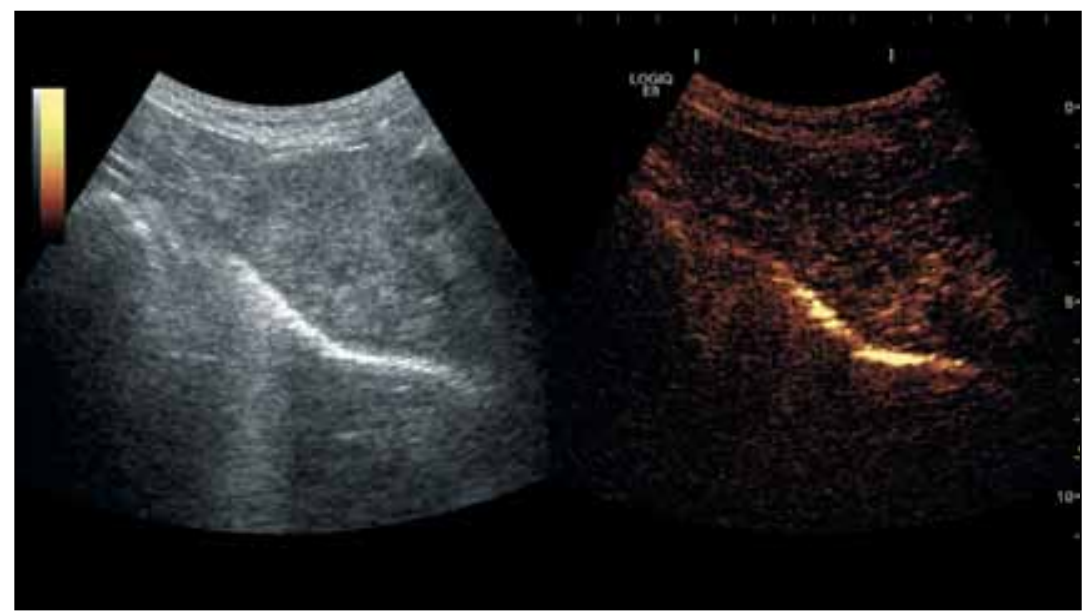

Fig. 3. Optimized conventional ultrasound with contrast agents highlights, the washing of the captured substance during the early venous stage.

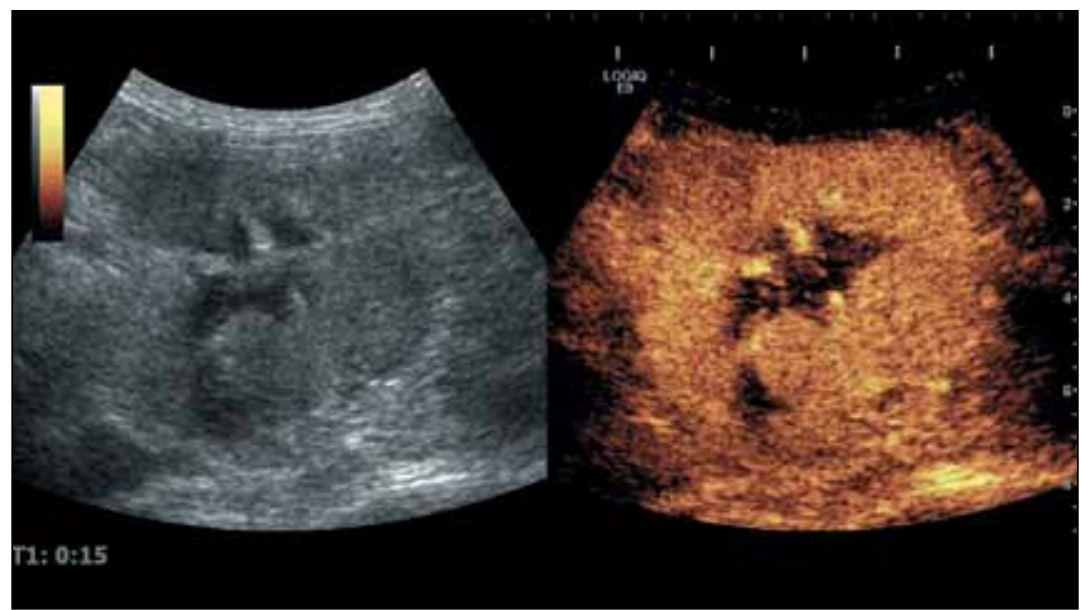

Fig. 4. Optimized conventional ultrasound with contrast material highlights impaired renal physiological structure by the presence of hypervascular formations, with intense capture in early arterial time. 
Plate IX

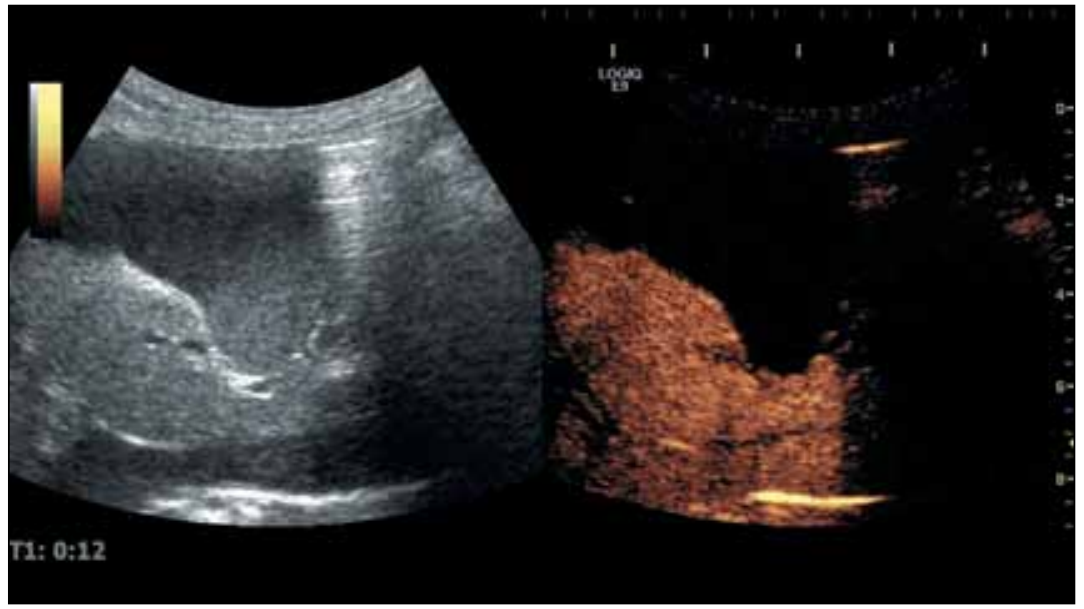

Fig. 5. Lack of pathological collection of the contrast substances in the gallbladder (*). Malign gallbladder sludge (arrow).

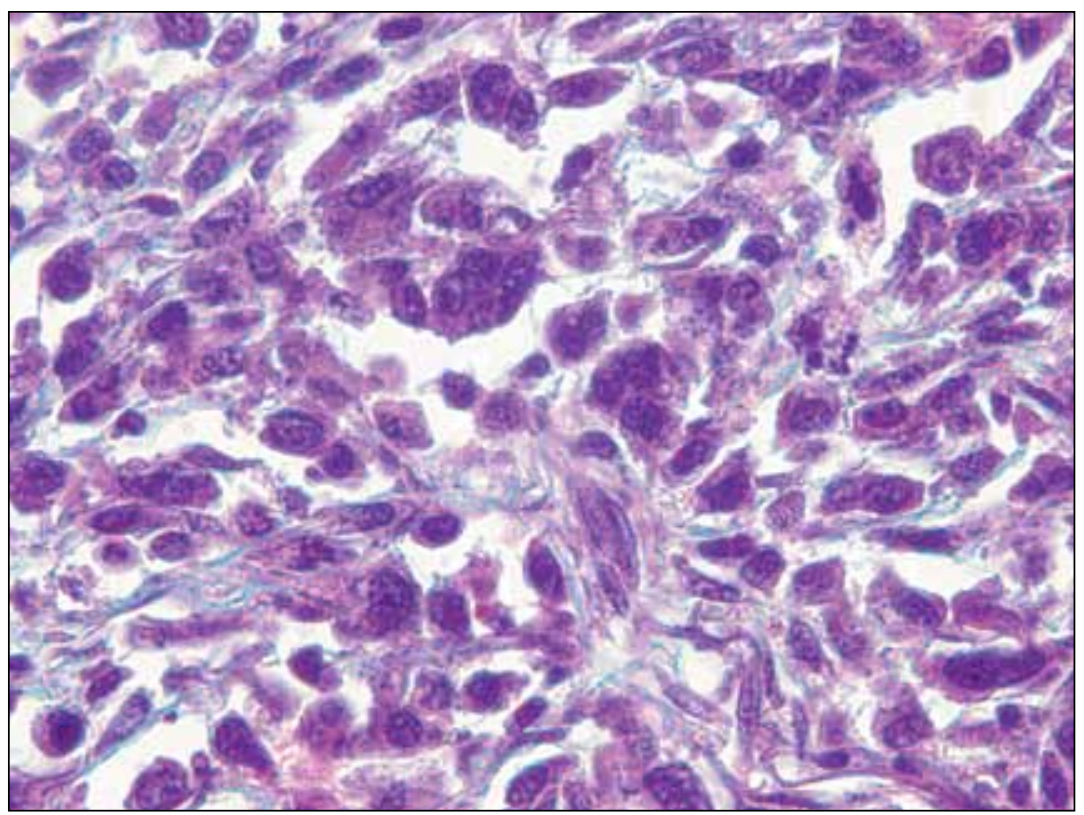

Fig. 6. Histology - anaplasic carcinoma of the prostate in dog. Transmission microscope, haematoxyline-eosine, magnification $\times 40$. 